ISSN 2637-7764

\title{
Incidental Finding of an Atypical Benign Fibro-Osseous Lesion in the Mandible of an Adolescent Orthodontic Patient
}

Henry JI, Newaz ZA and Jerrold LE*

NYU Langone Medical Center, USA

*Corresponding author: Laurance Jerrold, JD $21954^{\text {th }}$ St. Brooklyn, NY 11220, USA

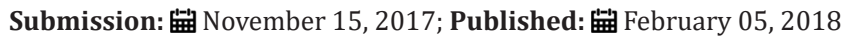

\begin{abstract}
This case report highlights the formation and progression of a lesion with fibro-osseous characteristics that developed incidentally in a young African American female orthodontic patient without symptoms or adverse sequelae. In addition to discussing clinic-radiographic correlation and differential diagnosis of the lesion, this report explains some considerations for management when encountering these findings.
\end{abstract}

Case Presentation

History and initial presentation

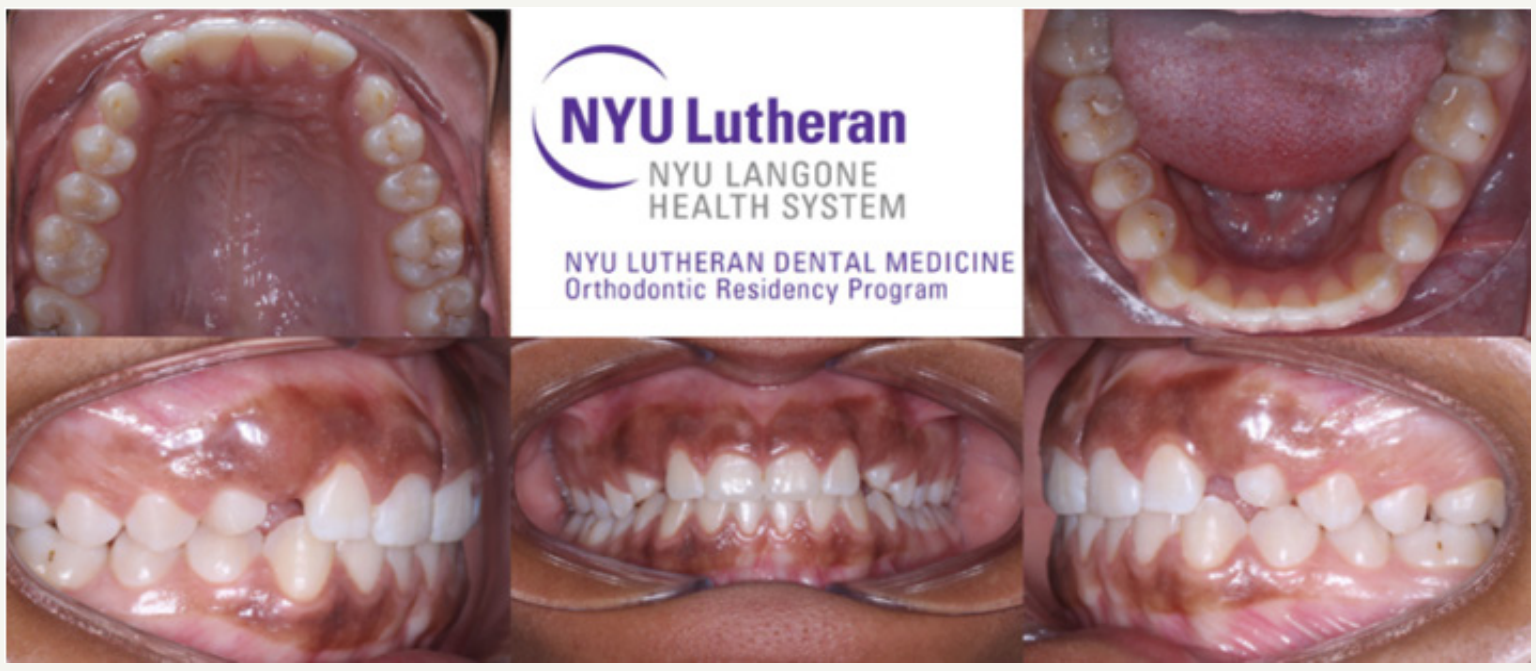

Figure 1: Intraoral photo composite from initial records - Note in the mandibular occlusal view that \#44 may be slightly extruded compared to adjacent teeth. The buccal right photo also implies minor extrusion of \#44, not seen in the premolars of the contra lateral occlusal plane.

A 15 year old African American female presented to NYU Langone Health-Brooklyn, for routine orthodontic records in 2016 (Figure 1). The patient had a noncontributory medical history, was not taking any medications, and reported no known allergies. The patient's chief complaint was, "the dentist sent us here because I might need to have some baby teeth extracted." Clinical exam revealed a Class III dental relationship, upper arch spacing in the canine region, over-retained upper primary canines, buccally palpable upper permanent canines, centered midlines, $2 \mathrm{~mm}$ of overbite and $1 \mathrm{~mm}$ of overjet, no TMJ symptoms, no functional shifts, and an obtuse nasolabial angle.

Radiographic findings, differential diagnosis, and clinical correlation

Routine panoramic and cephalometric radiographs were consistent with the clinical findings. Cephalometric evaluation 
revealed a slight skeletal class III tendency, normo divergent vertical growth pattern, and the upper and lower in cisor proclination was within normal limits. However, it was incidentally noted that there was a well-defined radiopaque mass in the body of the right mandible apical to the lower right first premolar \#44 (Figure 2). The lesion appeared to be associated with slight hyper occlusion of \#44, which was also clinically evident. The patient reported no current pain, discomfort, or paresthesia, and denied any history of such symptoms. To better evaluate specific characteristics of the lesion, a CBCT was acquired and a maxillofacial radiologist was consulted. The CBCT data better helped visualize the bucco lingual extent of the lesion, which extended from the buccal to the lingual cortical plate but without bony expansion. The internal architecture appeared to be a radiopaque mass with a ground-glass pattern and peripherally it was mostly surrounded by normal trabecular bone with hints of a thin relative radiolucent appearance in some areas (Figure 3). The disease category primarily suspected was the benign fibro-osseous category as the internal contents of the lesion did not resemble normal bony architectural features usually seen in cortical or trabecular bone. The differential diagnosis included peri apical cemento-osseous dysplasia, fibrous dysplasia (localized variant), cement oblastoma, and dense bone island (idiopathic osteosclerosis). Endodontic testing of teeth in the region was recommended. All teeth in the quadrant were pulp tested and were determined to be vital, including \#44. All teeth were also asymptomatic to percussion and palpation testing. We were able to acquire two prior panoramic radiographs for comparison (Figure 4) dating from six and four years prior to our current radiograph respectively. The 2010 radiograph did not reveal evidence of a lesion, but the 2012 film did exhibit the lesion, which appeared similar to that seen in 2016, but perhaps slightly lesser in size (Figure 5).

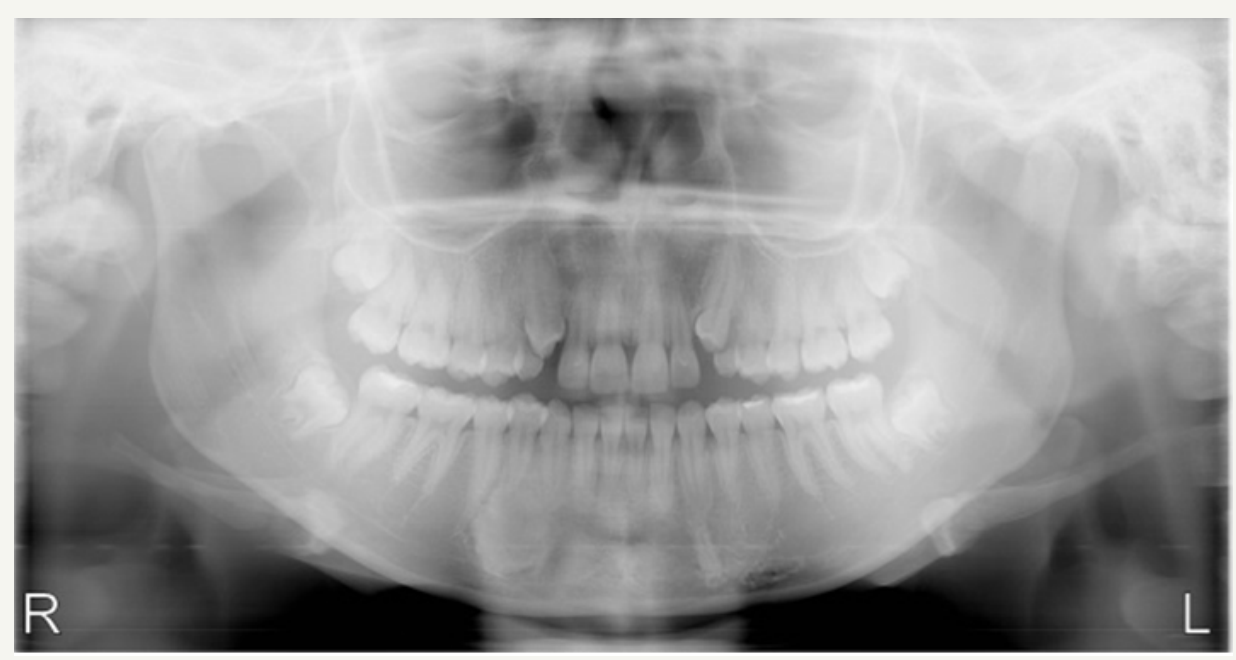

Figure 2: Panoramic image from initial records, July 2016.

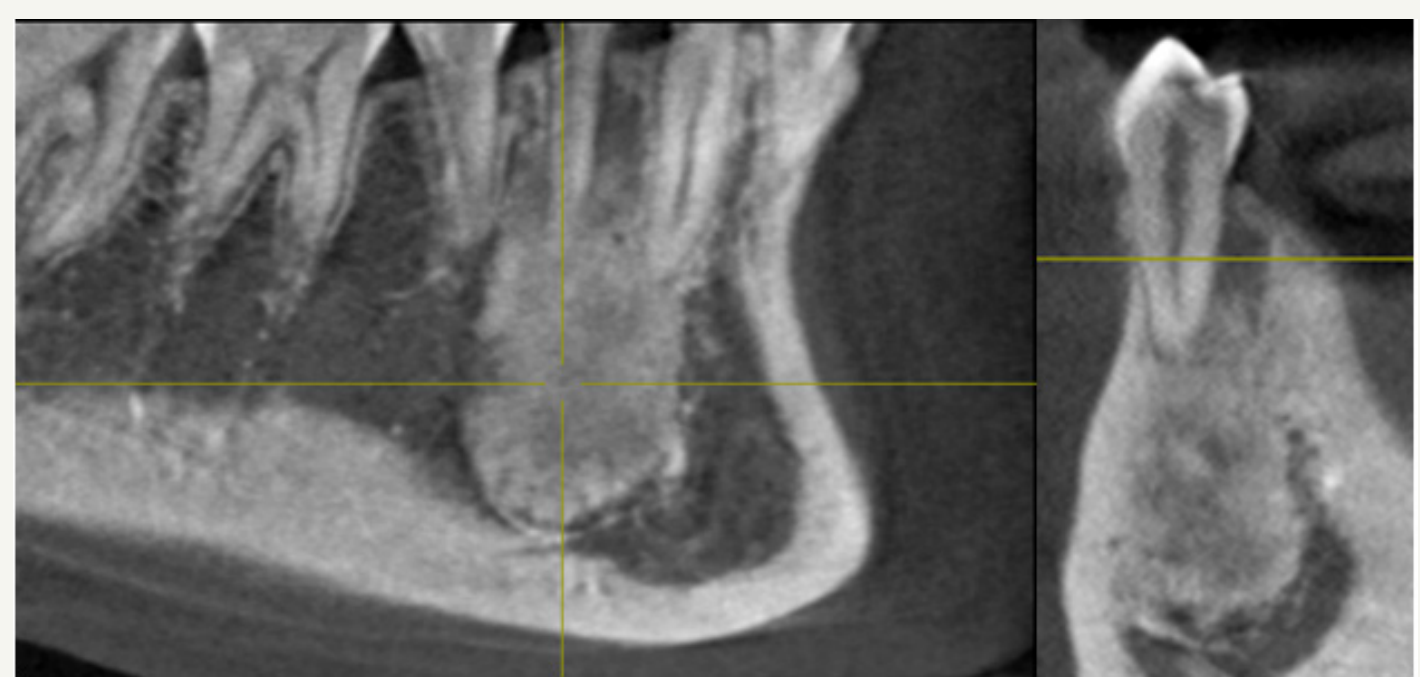

Figure 3: Selected CBCT views subsequent to initial records. Various depictions of the granular radio opacity apical to \#44 are provided showing the ground-glass radio opaque mass, with a relatively sclerotic periphery, and lack of a discernable soft tissue capsule. Although peripheral to the mass, it is relatively more radiolucent in some areas. The majority of the mass is directly surrounded by what appears to be normal bone. 

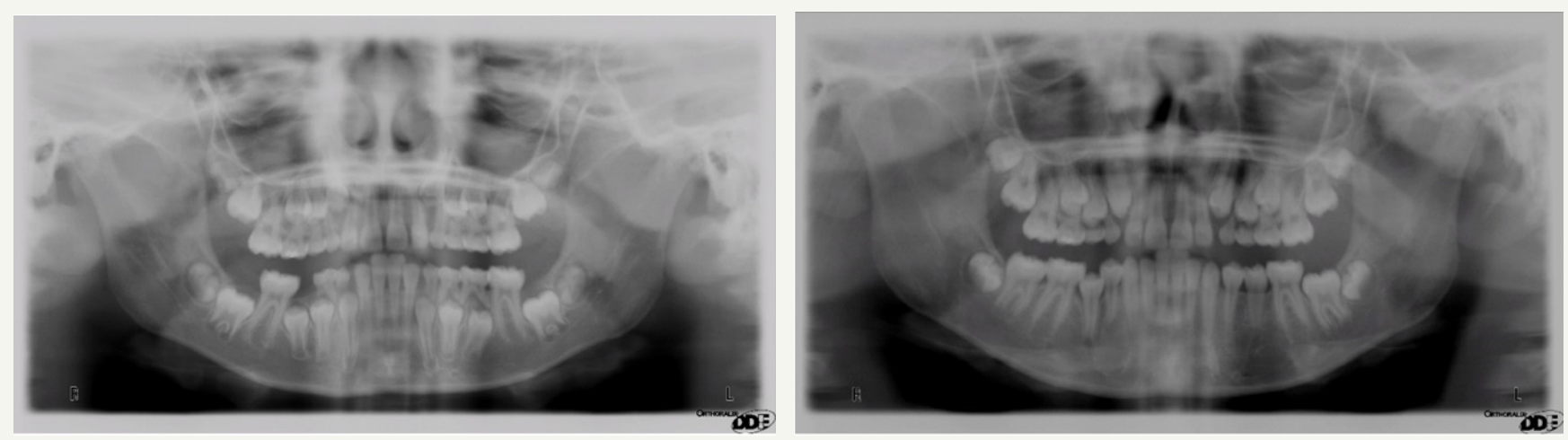

Figure 4: Prior radiographs from A) March 2010 and B) November 2012. Note that in (A), No discernable lesion was noted, and in (B), it can be appreciated. This suggests that the lesion primarily manifested sometime between age 9 and 11 although its subtle beginnings may have begun sooner. Retrospectively, the bone density and root separation between the mandibular canine and first premolar in (A) appears slightly different between the right and left sides.

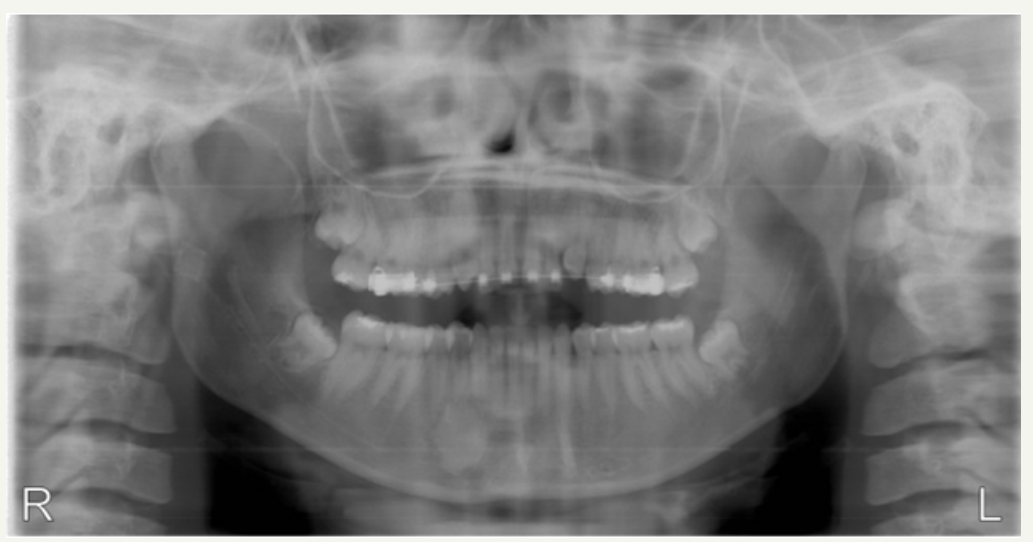

Figure 5: Follow-up radiograph acquired approximately one year after initial presentation to the orthodontic clinic - August 2017. Lack of gross changes suggests that the lesion likely has stabilized for the time being.

\section{Treatment and management}

Biopsy or other interventional treatment was notrecommended as the lesion did not exhibit adverse size or behavioral changes thus a definitive determination of the lesion's entity was problematic. Periapical osseous dysplasia was considered, but the ground-glass pattern, although having been reported, is uncommon for these lesions; they usually present as radiolucent lesions with a central opaque focus that gradually opacifies with age [1-3]. In a young patient, a more prominent radiolucent component would have been typical. Fibrous dysplasia was also considered, but the typical presentation is more diffuse, ill-defined, and is often expansile in nature, although localized varieties have been reported [1,2]. Cement oblastomas are usually associated with clinical evidence of pain, have a distinct soft tissue capsule circumferentially and often are accompanied by horizontal apical root resorption, which was not noted [1,2]. Dense bone islands typically have a density and "texture" approximating that of cortical bone, as opposed to the granular appearance seen in this patient's lesion [1]. With these considerations, the more likely entities included periapical osseous dysplasia and a localized variant of fibrous dysplasia. Diagnosis of these lesions is usually made radio graphically. Biopsy is not indicated for cemento-osseous dysplasia lesions as their reduced vascularity increases the risk of secondary infection in the surgical site. Fibrous dysplasia treatment varies based on extent, and lesions of a docile nature are typically not surgically examined. The preferred and more conservative method of management is to periodically monitor the lesion. Radio graphically, at regular 6 month recall intervals, and to consider a more aggressive interventional course of action only if gross changes became noticeable radiographically or if symptoms or clinical evidence of local change began to arise. It was deemed that orthodontic treatment could be initiated as planned. The orthodontic treatment plan consisted of extracting the over-retained upper primary canines, banding and bonding the upper arch only, exposing and ligating the upper permanent canines if needed, and closing any residual upper arch spacing as required to achieve the best Class I canine relationship possible. Treatment was commenced and progressed uneventfully. A subsequent follow-up panoramic radiograph revealed no gross changes in size or features from the initial panoramic records image and the early CBCT. Sufficient time has elapsed without gross radiographic changes to deem the lesion to be tentatively stable; radiographic monitoring will continue as appropriate.

\section{Conclusion}

The primary suspected entity for the lesion incidentally noted in the adolescent African American female patient was a benign fibro-osseous lesion, with features not pathognomonic for any one 
particular lesion in the differential diagnosis. Treatment consisted of an attempt to gain familiarity with the behavior of the lesions in question, and in collaboration with a maxillofacial radiologist, helped guide the decision to manage the lesion by closely monitoring it without surgical intervention.

\section{References}

1. White SC, Pharoah MJ (2014) In Oral Radiology-E-Book: Principles and Interpretation. Elsevier Health Sciences, USA.

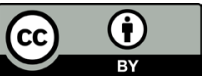

Creative Commons Attribution 4.0

International License

For possible submission use the below is the URL
2. Koenig LJ, Tamimi DF, Petrikowski GC, Perschbacher SE (2017) In Diagnostic Imaging: Oral and Maxillofacial. Elsevier Health Sciences Division, USA.

3. Alsufyani NA, Lam E (2011) Osseous (cemento-osseous) dysplasia of the jaws: clinical and radiographic analysis. J Can Dent Assoc 77: b70.

\section{Your subsequent submission with Crimson Publishers will attain the below benefits}

- High-level peer review and editorial services

- Freely accessible online immediately upon publication

- Authors retain the copyright to their work

- Licensing it under a Creative Commons license

- Visibility through different online platforms

- Global attainment for your research

- Article availability in different formats (Pdf, E-pub, Full Text)

- Endless customer service

- Reasonable Membership services

- Reprints availability upon request

- One step article tracking system 